\title{
AUTOCORRECTING RECONSTRUCTION FOR FLEXIBLE CT SCANNERS
}

\author{
Jeff Orchard* ${ }^{*}$ Alexei Ramotar ${ }^{\dagger}$ \\ David R. Cheriton School of Computer Science, University of Waterloo \\ \{jorchard,aramotar\}@cs.uwaterloo.ca
}

\begin{abstract}
Nanotechnology researchers are developing miniature, lowpower $\mathrm{x}$-ray devices. These innovations might revolutionize the world of computed tomography (CT). Tiny x-ray emitters and detectors could be embedded on a flexible sheet and deployed around a body part to acquire CT data at the scene of an accident. However, the irregular geometry of such a scanner makes the reconstruction problem more challenging. Moreover, measurement errors in the positions of the emitters and detectors would limit the quality of the resulting images. We propose a robust reconstruction methodology that can automatically correct for small errors in the CT scanner geometry. The method uses a simple optimization scheme to minimize the entropy of the reconstructed image. Test cases suggest that this is a viable approach to robust reconstruction for flexible CT scanners.
\end{abstract}

Index Terms: computed tomography, image reconstruction, entropy, nanotechnology, autofocus

\section{INTRODUCTION}

Advances in nanotechnology are paving the way for an exciting new breed of small, portable, flexible and versatile CT scanners that could be used by a paramedic at the scene of an accident. Researchers are developing minute (pixel-sized), low-power x-ray emitters and detectors [1]. Work is being done to integrate these tiny $\mathrm{x}$-ray devices into a flexible array [2]. When wrapped about a body part, the x-ray emitters can be individually pulsed in rapid succession to acquire raw CT data. However, along with these new CT devices come some interesting challenges.

Currently, all CT scanners use reconstruction methods that are specifically catered to their fixed geometry. Moreover, the acquisition geometry is often designed to make the reconstruction process efficient and accurate by producing data that is suitable for reconstruction by the popular filtered backprojection method or Fourier reconstruction method.

*J. Orchard was funded by the Natural Sciences and Engineering Research Council of Canada, and the Canadian Foundation for Innovation

${ }^{\dagger}$ A. Ramotar was funded by a Canadian Commonwealth Scholarship.
We expect that a portable, versatile CT scanner will not have the luxury of a fixed geometry, forcing the reconstruction methods to be of a more general flavour. The methods will have to be capable of using irregularly sampled data. Some approaches that can handle irregular geometry exist. For example, the CT reconstruction problem can be formulated as a linear system of equations. Unfortunately, the system is so big (over 65,000 variables for even a modest sized problem) that the system matrix alone will not fit into the memory of most desktop workstations. A way to avoid having to store the whole matrix is to use an iterative projection method such as Kaczmarz method [3]. These methods tend to be quite slow. Another approach is to use interpolation in the Radon Transform [4]. This is the approach that we take here.

We also expect that these bendable CT scanners will be susceptible to uncertainty in the locations of the x-ray emitters and detectors. The reconstruction process needs to know how the x-ray emitters and detectors are spatially distributed. We foresee the use of a motion-tracking device such as ShapeTape (Norther Digital Inc., Waterloo, Canada) to estimate the scanner geometry. However, these devices are prone to measurement errors. Correcting these geometrical errors is similar in nature to the problem of motion-correction in medical image reconstruction. An automatic (data-driven) motion-correction method for SPECT (single photon emission computed tomography) uses an iterative approach, repeatedly reconstructing the image based on a guess of the patient motion, and then uses that reconstruction to derive a better estimate of the motion [5]. In MRI, some methods use image entropy as a measure of motion artifact, and apply motion adjustments to minimize the entropy of the reconstructed MR images [6, 7].

In this paper, we present a method that minimizes the entropy of the reconstructed image to derive a correction to the CT geometry and achieve a nearly error-free reconstruction.

\section{METHODS}

For this flexible scanner, we will distinguish between two instances of its geometrical configuration. The acquisition geometry refers to the positions of the x-ray emitters and detectors when the data is acquired, whereas the reconstruction geometry refers to the positions of the $\mathrm{x}$-ray emitters and detectors that are used in the reconstruction. Ideally, the acqui- 


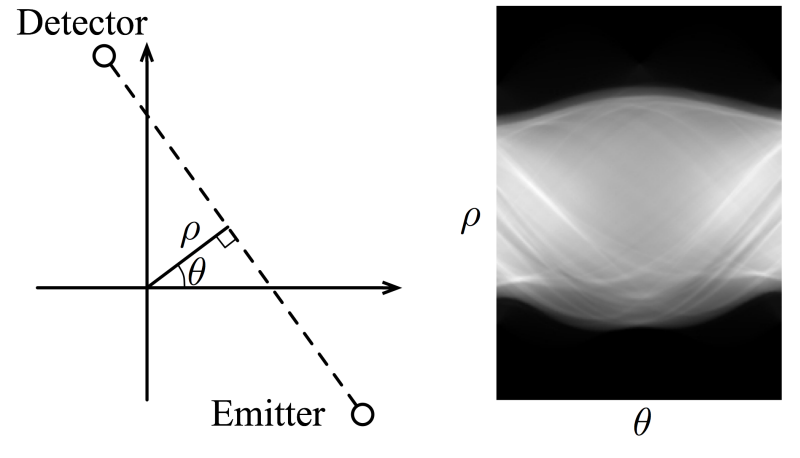

Fig. 1. Ray sum geometry and the Radon Transform

sition and reconstruction geometries are the same. However, measurement inaccuracies will likely cause the reconstruction geometry to differ from the true acquisition geometry. The first challenge is to devise an efficient reconstruction method for irregular acquisition geometries. The second challenge is to make that reconstruction method robust to differences between the acquisition and reconstruction geometries. That is, to perform autocorrecting or autofocussing.

\subsection{Reconstruction}

Since the scanner is flexible, we cannot directly take advantage or Fourier reconstruction of filtered backprojection, like most CT scanners. For the purpose of reconstruction, the acquisition geometry is assumed to be general, simply a collection of x-ray emitters and detectors scattered around the object to be imaged. Each emitter-detector pairing can potentially contribute a ray sum to the acquired data. Practically speaking, though, the emitters give off $\mathrm{x}$-rays in the form of a fan-beam centered on a particular direction, and only detectors that lie within that fan will actually collect a ray sum from that emitter. Thus, the data collected by the scanner is a set of ray sums that corresponds to a subset of the possible emitter-detector pairs.

Each ray sum, in essence, is the integral of the logarithm of the attenuation field through the object being imaged. As is standard in CT reconstruction, the line for each ray sum can be represented uniquely by the shortest line drawn from the origin to the ray, as shown in Fig. 2.1. As such, the ray can be represented as a point in $(\rho, \theta)$ space, where $\rho$ is the perpendicular distance from the ray to the scanner origin, and $\theta$ is the complement of the angle that the ray makes with the coordinate system's $x$-axis. The data represented in this $(\rho, \theta)$ space is called the Radon Transform.

Our process of reconstruction follows the method outlined in [4]. The method involves taking all the ray sums produced by the emitter/detector pairs, placing them into the $(\rho, \theta)$ space of the Radon Transform, and resampling them onto a regular grid. Once on a regular grid, a standard parallel- beam filtered backprojection method is employed to reconstruct the image. However, in [4], all the samples that fell within a half-pixel distance of a grid point were merely averaged to arrive at a value for the grid point. We have found that the Radon Transform more closely matches its parallel-beam analog if the data points are weighted according to the inverse of their distance from the resampling grid point. We used just such a weighted averaging for our reconstruction.

\subsection{Autofucussing}

We anticipate that the presence of errors in the reconstruction geometry will introduce artifacts in the reconstructed image, disrupting the crisp edges between materials (e.g. bone versus soft tissue versus air). Our hypothesis is that these artifacts will increase the image entropy by spreading out the image histogram. Entropy is minimized when an image has nearly uniform regions with well-defined boundaries. Thus, our autofocussing method seeks to adjust the reconstruction geometry in an effort to minimize the entropy of the reconstructed image.

\subsection{Experiments}

In our simulation experiments, we used two $256 \times 256$ pixel CT images from the Visible Human Project (National Library of Medicine): one head slice, and one slice from the pelvis. We placed (virtual) emitters and detectors on a circle around the object. The emitters were spaced one pixel distance apart on the circumference of the circle, with one detector placed between adjacent emitters. The ray sums were computed using a discrete approximation to the ray sum integral. The integration variable $t$ traversed the ray from the emitter to the detector. To approximate this integral in our simulations, we linearly interpolated the image along the ray line, taking samples every 0.3 pixel-lengths along the line. The value of the integral was the sum of those samples, divided by 0.3 .

With the idea in mind that the emitters and detectors will be embedded into a flexible band, their positions were displaced from the circular geometry in a coherent and smooth manner. Five control points were put evenly-spaced on the circle, but displaced to perturb the emitters and detectors from their circular geometry. Without loss of generality, one control point was kept fixed on the circle, leaving four other points to move freely. A periodic cubic spline was fit to each of the $x$ - and $y$-components of these displacements, yielding a parametric representation of a perturbation to the original circular geometry. This parametric curve was used to displace the emitters and detectors.

As mentioned earlier, there are two geometries that come into play during imaging: the (true) acquisition geometry, and the (assumed) reconstruction geometry. We used perturbations to generate geometrical configurations for each. That is, we used a perturbed version of the circular geometry as the 


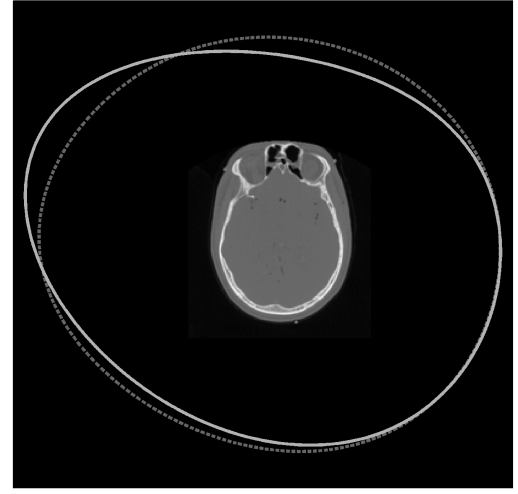

Fig. 2. Acquisition geometry (dashed line) and initial reconstruction geometry (solid line)

acquisition geometry, then perturbed the acquisition geometry to get our reconstruction geometry. Figure 2 shows both the acquisition and reconstruction geometries used for the CT head slice test. The control point displacements were formed from eight Gaussian-distributed random numbers (mean of zero, standard deviation of 12.8 pixels).

Starting from the erroneous reconstruction geometry, we used the Nelder-Mead simplex method (a variant of Matlab's fminsearch function submitted by Olivier Salvado) to adjust the reconstruction geometry until the image entropy was minimized. Image entropy was calculated using 128 bins covering the intensity range $[0,255]$. Just like in the previous geometrical perturbations, the geometry was adjusted using eight numbers representing the $x$ - and $y$-displacements of four control points (with a fifth control point held fixed). During each geometrical perturbation, the total perimeter of the emitter/detector loop was forced to remain constant to prevent the geometry from collapsing inward.

\section{RESULTS}

Figure 3 shows how the entropy changes with varying degrees of error in the reconstruction geometry. The sharp downward cusp at zero displacement (i.e. no errors in the reconstruction geometry) indicates that image entropy is a sensitive measure of errors in the reconstruction geometry. While the plot shows the behaviour of the cost function along only a single direction through the parameter space, we consistently observe a similar curve using randomly-generated geometrical perturbations, which leads one to believe that entropy is an effective measure for this autofocussing task.

Reconstructions using our method are shown in Fig. 4. The original CT image has an entropy of 1.73 , and is shown in (a). Figure 4(b) shows the reconstruction using the correct geometry (i.e. the reconstruction geometry equals the acquisition geometry). It is worth noting that the resampling

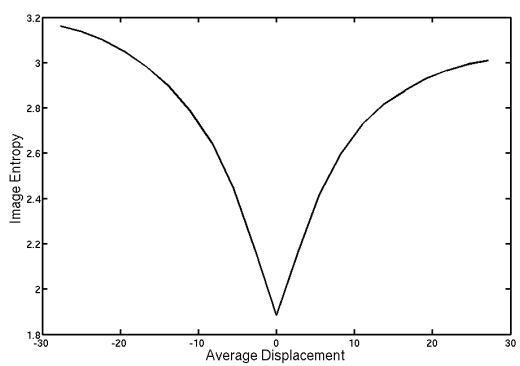

Fig. 3. Image entropy as a function of error in the reconstruction geometry. The horizontal axis charts the average displacement (in pixels) for the emitters and detectors from their positions in the acquisition geometry, with negative values indicating a displacement in the direction opposite the corresponding positive values.

process of gridding the Radon Transform data constitutes a fundamental loss of information, resulting in a slight blurring of the reconstructed image. The entropy of the correctly reconstructed image shown in (b) is 1.88. Figure 4(c) shows the image that results if the erroneous reconstruction geometry is used to reconstruction the image. Notice the smudge-like artifacts that obfuscate many of the tissue boundaries. Its entropy is 2.53. The result from the autofocussing method is shown in Fig. 4(d), yielding an entropy of 1.97 .

Similar results were observed using the CT pelvis image. The original image had an entropy of 2.00 , while the reconstruction without any geometrical error had an entropy of 2.06. The reconstructed image using the erroneous reconstruction geometry was 3.32 , while the autocorrected reconstruction had an entropy of 2.16 , and was visually indistinguishable from the reconstruction that used the error-free geometry.

The running time for the autocorrection procedure was 329 seconds on an Apple Macintosh Dual 2.5GHz PowerPC G5 workstation with 8GB of RAM (one processor was disabled for this benchmark). The method was implemented using Matlab R2006b (MathWorks Inc., Natick, Massachusetts). Some of the most CPU intense portions of the code were optimized and compiled using Matlab's mex facility. For example, the backprojection procedure was implemented in $\mathrm{C}$ code, making Matlab's iradon run six times faster (This accelerated version of iradon can be downloaded from the Matlab Central File Exchange).

\section{CONCLUSIONS AND FUTURE WORK}

We have shown that image entropy is a viable measure of image artifacts caused by error in the reconstruction geometry, with a well-defined minimum value that seems to correspond to the correct reconstruction. This cost function also seems to be smooth and consistent enough to guide a simple optimiza- 


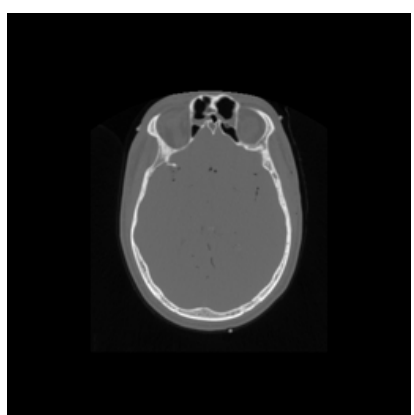

(a) Original, $E=1.73$

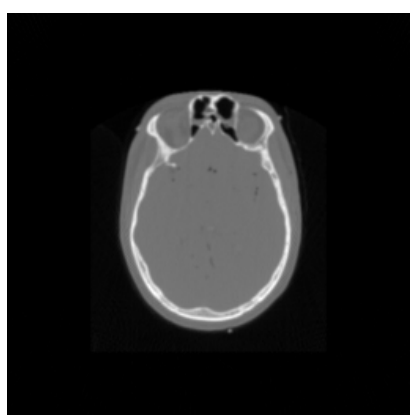

(b) Error Free Recon, $E=1.88$

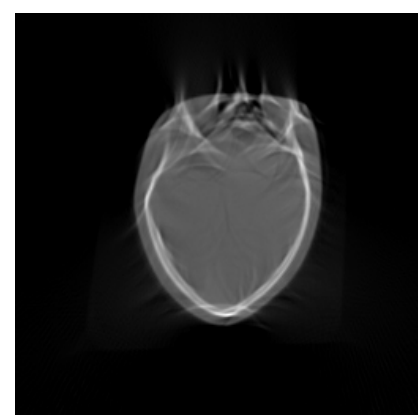

(c) Uncorrected Recon, $E=2.53$

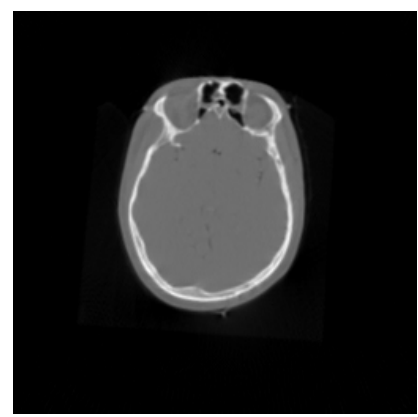

(d) Autofucussed Recon, $E=1.97$

Fig. 4. Original $256 \times 256 \mathrm{CT}$ head image, along with various reconstructions

tion method to the desired solution.

One direction for future development is to implement a more mathematically-inspired resampling method for the regridding step of the Radon Transform. A sinc interpolation strategy might be appropriate, or perhaps we should pose the resampling procedure as the solution of a linear system of equations, such as in [8]. Given the results in this study, though, these methods would have to have efficient implementations to make them worthwhile.

The eventual materialization of this device will most likely involve 3D scanning since it is unrealistic to hope that the emitters and detectors will stay in the same plane. Extending this method to 3D seems straightforward, but nonetheless calls for investigation.

These results are promising, but a more complete study is necessary to determine the method's capabilities and limitations. In particular, it is important to investigate how many degrees of freedom the optimization scheme can tolerate, and what the running time might be in a realistic scenario.

\section{REFERENCES}

[1] G. Z. Yue, Q. Qiu, B. Gao, Y. Cheng, J. Zhang, H. Shimoda, S. Chang, J. P. Lu, and O. Zhou, "Generation of continuous and pulsed diagnostic imaging x-ray radiation using a carbon-nanotube-based field-emission cathode," Applied Physics Letters, vol. 81, no. 2, pp. 355-357, July 2002.

[2] Niraj Sinha and John T.-W. Yeow, "Carbon nanotubes for biomedical applications," IEEE Transactions on Nanobioscience, vol. 4, no. 3, pp. 180-195, June 2005.
[3] Charles L. Epstein, Mathematics of Medical Imaging, Prentice Hall, Upper Saddle River, NJ, 2003.

[4] Alexei Ramotar and Jeff Orchard, "General geometry CT reconstruction," in Proc. of the International Conference on Image Processing and Computer Vision (IPCV'06), 2006, pp. 95-99.

[5] Andre Z. Kyme, Brian F. Hutton, Rochelle L. Hatton, David W. Skerrett, and Leighton R. Barnden, "Practical aspects of a data-driven motion correction approach for brain SPECT," IEEE Trans. Med. Imag., vol. 22, no. 6, pp. 722-729, 2003.

[6] David Atkinson and Derek L. G. Hill, "Automatic correction of motion artifacts in magnetic resonance images using an entropy focus criterion," IEEE Trans. Med. Imag., vol. 16, no. 6, pp. 903-910, 1997.

[7] Armando Manduca, Kiaran P. McGee, E. Brian Welch, Joel P. Felmlee, and Richard L. Ehman, "Fast automatic correction of motion artifacts in shoulder MRI," in Proceedings of SPIE Medical Imaging, San Diego, 2001, pp. 853-859.

[8] David Atkinson, David A. Porter, Derek L. G. Hill, Fernando Calamante, and Alan Connelly, "Sampling and reconstruction effects due to motion in diffusion-weighted interleaved echo planar imaging," Magn. Reson. Med., vol. 44, pp. 101-109, 2000. 